\title{
How severe COVID-19 infection is changing ARDS management
}

\author{
Niall D. Ferguson ${ }^{1,2,3,4^{*}}$ (D) Tài Pham ${ }^{5,6,7}$ (iD and Michelle Ng Gong 8,9
}

(0) 2020 Springer-Verlag GmbH Germany, part of Springer Nature

\section{Plus ça change, plus c'est la même chose....}

Over the last 6 months intensivists and non-intensivists around the world have been treating patients with acute respiratory distress syndrome (ARDS) brought on by COVID-19, often in extreme conditions with overwhelmed healthcare systems. As the first wave of the pandemic has passed in Europe and continues to progress in parts of North America, we pause to consider how severe COVID-19 infection is changing ARDS management and what the lasting implications might be for ARDS from other causes (Table 1).

Our first thought is that COVID-19 is changing everything and nothing about ARDS management. Everything, in the sense that the thousands of severe COVID-19 patients have brought widespread attention from nonintensivists and the general public to the high mortality and management challenges of ARDS. This is refreshing as prior to the pandemic, ARDS was often under-recognized, even among the intensivists who cared for such patients [1]. But at the same time, nothing has changed as all of these 'new-found' therapies and supportive techniques are not actually new-they just have not been as well understood, applied or implemented before the pandemic.

The foundation to ARDS management has been meticulous supportive care such as low tidal volume ventilation, and prone positioning in moderate-severe ARDS, both of which have been shown to reduce mortality. While proning had been reasonably well adopted in many European centres, its uptake in North America was poor, even in academic teaching centres, ranging from 8 to $15 \%$

\footnotetext{
*Correspondence: n.ferguson@utoronto.ca

${ }^{4}$ Toronto General Hospital Research Institute, 585 University Ave, 11-PMB-120, Toronto, ON M5G 2N2, Canada

Full author information is available at the end of the article
}

of moderate-severe ARDS patients [1, 2]. Common reasons for deciding not to prone include a lack of comfort with the procedure, misconceptions that the patient may not be hypoxemic enough, and concerns about hemodynamics [3]. With COVID-19 surges, the large number of severely hypoxemic patients forced many intensive care units (ICUs) to discover that they can indeed provide care in the prone position for moderate-severe ARDS patients. Most centres have trained staff in how to prone and many have developed dedicated proning teams to facilitate this care [4]. Indeed, interest in proning COVID-19 patients has extended to non-intubated awake patients, [5] and proning of intubated and nonintubated patients has even been recognized in mainstream media (https://www.nytimes.com/2020/05/13/ health/coronavirus-proning-lungs.html). Even after the COVID-19 surge has passed, we are hopeful that the future threshold for proning in ARDS will remain much lower than it was in 2019.

ARDS is known to be a heterogeneous syndrome with different sub-phenotypes that are characterized by different clinical features, inflammatory cytokine profiles, physiology and differential response to interventions [6, 7]. COVID-19 is no exception to this rule. Indeed, the large number of simultaneous patients with the same underlying etiology but varying physiological responses has put the importance of adapting mechanical ventilation strategies to the individual patient into sharp focus. One positive effect of clinical debates that have raged in the pages of medical journals and on Twitter is that respiratory mechanics are cool again. Many more clinicians are interested in the basics such as plateau pressure, respiratory system compliance and driving pressure, with others still going beyond that and considering airway opening pressure and recruitment to inflation ratio, [8] occlusion pressure, [9] and transpulmonary pressures [10]. While we hope this will

\section{Springer}


Table 1 How severe COVID-19 is changing ARDS management

\begin{tabular}{|c|c|}
\hline Paradigm & Example \\
\hline $\begin{array}{l}\text { Increased adoption of therapies previously shown to be effective in non- } \\
\text { COVID-19 ARDS }\end{array}$ & Prone positioning in moderate-severe ARDS \\
\hline Spillover adoption of therapies shown to be effective in COVID-19 ARDS & Early low-dose corticosteroids \\
\hline Applying evidence-based practice, informed by bedside physiology & Increased clinical interest in respiratory mechanics \\
\hline $\begin{array}{l}\text { Expanding new lines of investigation that may be relevant to both COVID- } \\
19 \text { and non-COVID-19 ARDS }\end{array}$ & $\begin{array}{l}\text { Systemic anticoagulation and anti-platelet agents for pulmonary vascular } \\
\text { thrombosis } \\
\text { Anti-inflammatory agents } \\
\text { Sedation with volatile anaesthetic agents } \\
\text { Stromal cell therapies }\end{array}$ \\
\hline $\begin{array}{l}\text { Highlighting the existence and importance of heterogeneity of treatment } \\
\text { effect in ARDS for many therapies }\end{array}$ & $\begin{array}{l}\text { Differential effects of steroids by severity } \\
\text { Proposals for several methods to individualize therapies (sub-phenotypes) }\end{array}$ \\
\hline Socializing randomization as the norm in critical care settings & Successful implementation of RECOVERY, REMAP-CAP, ACTIV platform trials \\
\hline
\end{tabular}

continue post-COVID-19, ongoing education, supervision and quality control will be necessary, as more complicated monitoring techniques may provide misleading information if improperly performed or interpretedcautionary tale of the pulmonary artery catheter.

Similarly, corticosteroids and other anti-inflammatory agents have been a source of controversy in ARDS and sepsis for more than 30 years with ongoing questions about which patients, if any, would most benefit. A number of randomized clinical trials have promised benefit of steroids over the years, though these have been small and often single-centre in nature. A recent multi-centre Spanish trial (Dexa) showed a mortality benefit with early dexamethasone in patients with persistent ARDS [11]. This was consistent with a growing body of literature showing benefit for steroids in severe community-acquired pneumonia. Pre-pandemic, corticosteroids were not routinely administered, however, because of both concerns about side-effects and ongoing uncertainty of their benefit. An order of magnitude larger than the previous largest trial, the preliminary report from the RECOVERY trial convincingly shows that early low-dose dexamethasone improves survival in patients with COVID-19, but heterogeneity of treatment effect is again appreciated with the largest benefit seen among those on mechanical ventilation, while those with no supplemental oxygen requirement did not benefit [12]. While we suspect that we will see a significant increase in corticosteroid use in early nonCOVID-19 ARDS, we hope that there will be additional studies in these patients both to determine short-term efficacy, as the mechanism of action may be different in COVID-19, and to examine long-term outcomes like ICU-acquired weakness.

It is becoming clear that many patients with COVID19 are in a hypercoagulable state and a number of reports show thrombosis and endothelial injury. This may account for some of the less typical ARDS presentations and has fueled debate about whether COVID-19 ARDS is actually ARDS [13]. However, inflammation, endothelial injury and pulmonary intravascular coagulation are common in ARDS [14]. Many patients with ARDS from COVID-19 display huge physiological deadspace, right ventricle dysfunction, and very high ventilatory ratios. However, we know that these findings are also prevalent in non-COVID-19 ARDS and are associated with increased mortality [15]. Prophylactic anticoagulation has always been part of the management in ARDS and other ICU patients. With ongoing trials pending, it remains to be seen whether higher doses of anticoagulation and anti-platelet agents would benefit ARDS patients from COVID or any other causes.

This pandemic has provided many clear examples of what not to do when it comes to generating new knowledge and integrating this into practice - from uncontrolled case series, opinion being touted as evidence in high impact journals, retracted studies, and questionable medical advice via Twitter and TikTok. These in themselves provide lessons in retrospect, but perhaps the most positive influence that the pandemic could have on future ARDS management is to socialize and normalize enrolment in randomized trials in the intensive care environment. The creation and success of ongoing platform trials such as RECOVERY, REMAP-CAP, and ACTIV during the pandemic will hopefully spur clinicians, patients and their families, regulators, funders, and research ethics boards to demand enrolment into randomized clinical trials, [16] as is commonly the case in the cancer field.

The COVID-19 pandemic has provided many intensivists (and some non-intensivists) several years' worth of severe ARDS management experience over the course of just a few months. While the challenges have, in places, been extreme, we hope that this experience will benefit 
future ARDS patients for years to come, even when the COVID-19 pandemic is for the history books.

\begin{abstract}
Author details
${ }^{1}$ Interdepartmental Division of Critical Care Medicine, University of Toronto, Toronto, Canada. ${ }^{2}$ Departments of Medicine, Physiology, Institute for Health Policy, Management and Evaluation, University of Toronto, Toronto, Canada. ${ }^{3}$ Department of Medicine, Division of Respirology and Critical Care, University Health Network and Sinai Health, Toronto, Canada. ${ }^{4}$ Toronto General Hospital Research Institute, 585 University Ave, 11-PMB-120, Toronto, ON M5G 2N2, Canada. ${ }^{5}$ Service de Médecine Intensive-Réanimation, AP-HP, Hôpital de Bicêtre, Hôpitaux Universitaires Paris-Saclay, Le Kremlin-Bicêtre, France. ${ }^{6}$ Faculté de Médecine, Université Paris-Saclay, Le Kremlin-Bicêtre, France. ${ }^{7}$ Équipe d'Épidémiologie Respiratoire Intégrative, Center for Epidemiology and Population Health (CESP), Université Paris-Saclay, UVSQ, Univ. Paris-Sud, Inserm, Villejuif, France. ${ }^{8}$ Division of Critical Care Medicine, Division of Pulmonary Medicine, Department of Medicine, Montefiore Medical Center, Albert Einstein College of Medicine, New York, NY, USA. ${ }^{9}$ Department of Epidemiology and Population Health, Albert Einstein College of Medicine, New York, NY, USA.
\end{abstract}

\section{Compliance with ethical standards}

\section{Conflicts of interest}

Dr. Ferguson reports personal fees from Xenios and Getinge, outside the submitted work.

\section{Publisher's Note}

Springer Nature remains neutral with regard to jurisdictional claims in published maps and institutional affiliations.

Received: 2 August 2020 Accepted: 9 September 2020

Published online: 18 September 2020

\section{References}

1. Bellani G et al (2016) Epidemiology, patterns of care, and mortality for patients with acute respiratory distress syndrome in intensive care units in 50 countries. JAMA 315:788-800

2. The National Heart Lung, and Blood Institute PETAL Clinical Trials Network (2019) Early neuromuscular blockade in the acute respiratory distress syndrome. New Engl J Med 380:1997-2008
3. Guérin C et al (2018) A prospective international observational prevalence study on prone positioning of ARDS patients: the APRONET (ARDS Prone Position Network) study. Intensive Care Med 44:22-37

4. Cummings MJ et al (2020) Epidemiology, clinical course, and outcomes of critically ill adults with COVID-19 in New York City: a prospective cohort study. Lancet. https://doi.org/10.1016/s0140-6736(20)31189-2

5. Coppo A et al (2020) Feasibility and physiological effects of prone positioning in non-intubated patients with acute respiratory failure due to COVID-19 (PRON-COVID): a prospective cohort study. Lancet Respir Med. https://doi.org/10.1016/s2213-2600(20)30268-x

6. Sinha P, Churpek MM, Calfee CS (2020) Machine learning classifier models can identify ARDS phenotypes using readily available clinical data. Am J RespCrit Care. https://doi.org/10.1164/rccm.202002-0347oc

7. Shankar-Hari M, Fan E, Ferguson ND (2018) Acute respiratory distress syndrome (ARDS) phenotyping. Intensive Care Med. https://doi.org/10.1007/ s00134-018-5480-6

8. Chen $\mathrm{L}$ et al (2020) Potential for lung recruitment estimated by the recruitment-to-inflation ratio in acute respiratory distress syndrome. Am J RespCrit Care 201:178-187

9. Bertoni $\mathrm{M}$ et al (2019) A novel non-invasive method to detect excessively high respiratory effort and dynamic transpulmonary driving pressure during mechanical ventilation. Crit Care 23:346

10. Mauri T et al (2016) Esophageal and transpulmonary pressure in the clinical setting: meaning, usefulness and perspectives. Intens Care Med 42:1360-1373

11. Villar J et al (2020) Dexamethasone treatment for the acute respiratory distress syndrome: a multicentre, randomised controlled trial. Lancet Respir Med. https://doi.org/10.1016/s2213-2600(19)30417-5

12. Recovery Collaborative Group (2020) Dexamethasone in hospitalized patients with Covid-19-preliminary report. New Engl J Med. https://doi. org/10.1056/nejmoa2021436

13. Marini JJ, Dellinger RP, Brodie D (2020) Integrating the evidence: confronting the COVID-19 elephant. Intens Care Med. https://doi.org/10.1007/ s00134-020-06195-z

14. Tomashefski JF (2000) Pulmonary pathology of acute respiratory distress syndrome. Clin Chest Med 21:435-466

15. Sinha P et al (2019) Physiologic analysis and clinical performance of the ventilatory ratio in acute respiratory distress syndrome. Am J RespCrit Care. https://doi.org/10.1164/rccm.201804-0692oc

16. Califf RM, Hernandez AF, Landray M (2020) Weighing the benefits and risks of proliferating observational treatment assessments observational cacophony randomized harmony. JAMA. https://doi.org/10.1001/ jama.2020.13319 